\title{
Sensitivity Analysis for the Inverse Problems of Electromagnetic Nondestructive Evaluation
}

\author{
Sándor BILICZ ${ }^{1}$ \\ Budapest University of Technology and Economics, Hungary
}

\begin{abstract}
Sensitivity analysis of the model-based inverse problem associated to electromagnetic nondestructive evaluation is dealt with. Some uncertainty of the arrangement is inevitable present (imprecise host material parameters, sensor mispositioning, etc.), and this induces uncertainty on the reconstructed defect parameters. The aim of this work is to present a methodology for the ranking of the different sources of random error according to their contribution to the reconstruction uncertainty. To this end, state-of-art mathematical tools of sensitivity analysis are applied, including Sobol' indices, and a polynomial chaos expansion surrogate model to reduce the computational burden of the method. A numerical example drawn from magnetic flux leakage nondestructive evaluation is presented to illustrate the proposed methodology.
\end{abstract}

Keywords. inverse problem, sensitivity analysis, Sobol' index, polynomial chaos, surrogate model

\section{Introduction}

Inverse problems of electromagnetic nondestructive evaluation (EM NDE) are dealt with in this work. Model-based inversion is to reconstruct defect parameters based on the measured EM field. This problem is usually traced back to an optimization problem for which various efficient global optimization algorithms have been applied for decades. However, beyond the mere reconstruction of defect parameters, the uncertainty of the parameter values obtained by the inversion algorithm is also important. This uncertainty is inevitably present because the setup parameters (e.g., host material, specimen dimensions, sensor position, etc.) are never precisely known.

Uncertainty quantification (UQ) is to assess the uncertainty induced on a quantity of interests due to random inputs: in our case, uncertainty of reconstructed defect parameters due to imprecise setup parameters. Sensitivity analysis aims at ranking the sources of uncertainty with respect to their contribution to the output uncertainty.

In the present contribution, a framework is proposed for the sensitivity analysis of the solution of the entire inverse problem. For certain values of the measured data, optimization-based inversion is performed to obtain the reconstructed defect parameters which obviously depends on the uncertain setup parameters. The quantitative character-

\footnotetext{
${ }^{1}$ Corresponding Author: Sándor Bilicz; E-mail: bilicz@evt.bme.hu
} 
ization of the uncertainty is given by the Sobol' indices [1,2], which quantify not only the contribution of each setup parameter but also of their combinations. The benefit from this sensitivity analysis is that one gains proper knowledge on how the uncertain setup parameters influence the reconstruction capability of an inversion procedure. This may promote the optimal design of NDE arrangements with respect to robustness. Furthermore, it can screen the most important sources of uncertainty, which have to be taken into account in another inversion routines, e.g., the model-free approach in [3]. Finally, the proposed method provides a deeper insight into the importance of different physical phenomena behind the EM NDE method.

\section{Problem definition}

Let the deterministic vector-vector function $\boldsymbol{y}=\boldsymbol{f}(\boldsymbol{x} \mid \boldsymbol{w})$ represent the relationship between the observable signal $y \in \mathbb{R}^{K}$ (e.g., spatial scan of the B-field in flux leakage testing), the vector of the defect parameters $x \in \mathbb{R}^{N}$ (e.g., crack dimensions) and the setup parameters $\boldsymbol{w} \in \mathbb{R}^{M}$ (e.g., host material permeability, probe misalignment, etc.). The forward operator $f$ is realized by electromagnetic simulation.

In recent works, the application of sensitivity analysis by means of Sobol' indices of the forward operator $f$ is presented [4]. Such studies can lead to valuable knowledge on (i) how each setup parameter $w_{i}$ contributes to the output uncertainty and (ii) whether the setup uncertainty hinders the reconstruction of small variations of the defect parameters.

In the present work, the inverse problem is considered as follows. For a certain output signal $\boldsymbol{y}^{\prime}$ resulted from a measurement, the inverse problem reads as

$$
\hat{\boldsymbol{x}}=\boldsymbol{g}(\boldsymbol{w}) \equiv \underset{\boldsymbol{x} \in \mathscr{X}}{\arg \min }\left\|\boldsymbol{y}^{\prime}-\boldsymbol{f}(\boldsymbol{x} \mid \boldsymbol{w})\right\|^{2}
$$

where $\mathscr{X}$ is the region of interest of the defect parameters and $\hat{\boldsymbol{x}}$ stands for the reconstructed defect parameter vector. The latter depends on the uncertain setup parameters $\boldsymbol{w}$, which are modelled by the random vector $W \in \mathbb{R}^{M}$. Hereafter the joint probability density function of $\boldsymbol{W}$ is assumed to be known (e.g., from manufacturing tolerances or based on a statistical estimation from measurements). The reconstructed defect parameters are also considered as a random vector $\hat{\boldsymbol{X}}=\boldsymbol{g}(\boldsymbol{W})$. The goal of the analysis presented below is twofold: (i) characterization of the mean and standard deviation of $\hat{\boldsymbol{X}}$, and (ii) calculation of the Sobol' indices of each component of $\hat{\boldsymbol{X}}$ with respect to the random setup parameters $\boldsymbol{W}$.

\section{Uncertainty quantification and sensitivity analysis}

To achieve the goals in the previous section, the following scheme is proposed.

1. A defect $\boldsymbol{x}^{\prime}$ is chosen and the inverse problem of reconstructing $\boldsymbol{x}^{\prime}$ from the corresponding measured output signal is studied. Note that different defects within $\mathscr{X}$ may yield different outcomes of the sensitivity analysis.

2. The measured output signal is approximated by the synthetic data $\boldsymbol{y}^{\prime}=\boldsymbol{f}\left(\boldsymbol{x}^{\prime} \mid \boldsymbol{w}\right)$, by using the deterministic forward model $f$. 
3. Finally, a heuristic approximation for $\boldsymbol{g}(\boldsymbol{w})$ is introduced as

$$
\boldsymbol{g}(\boldsymbol{w}) \approx \underset{\boldsymbol{x} \in \mathscr{X}}{\arg \min }\|\underbrace{\boldsymbol{f ( \boldsymbol { x } ^ { \prime } | \boldsymbol { w } )}}_{\boldsymbol{y}^{\prime}}-\underbrace{\boldsymbol{f}\left(\boldsymbol{x} \mid \boldsymbol{w}_{0}\right)}_{\tilde{\boldsymbol{f}}(\boldsymbol{x})}\|^{2}
$$

where $\boldsymbol{w}_{0}$ stands for the mean of the random setup parameters: $\boldsymbol{w}_{0}=\mathbb{E}[\boldsymbol{W}]$. This approximation reduces the computational cost of the optimization: the forward model with $\boldsymbol{w}=\boldsymbol{w}_{0}$ kept fixed, whereas $\boldsymbol{f}\left(\boldsymbol{x} \mid \boldsymbol{w}_{0}\right)$ can be approximated by a surrogate model $\tilde{\boldsymbol{f}}(\boldsymbol{x})$. The latter can be, e.g., a sparse grid [5], a kriging or a radial basis function [6] surrogate model, just to name a few among the common choices.

Sobol' indices. A state-of-art tool of sensitivity analysis is based on the Sobol' indices [2]. Each component (with notation omitted for simplicity) of the function $\boldsymbol{g}$ in Eq. (2) can be decomposed [1] as

$$
g(\boldsymbol{w})=g_{0}+\sum_{i=1}^{M} g_{i}\left(w_{i}\right)+\sum_{1 \leq i<j \leq M}^{M} g_{i j}\left(w_{i}, w_{j}\right)+\cdots+g_{1,2, \ldots, M}\left(w_{1}, w_{2}, \ldots, w_{M}\right),
$$

This decomposition is unique under certain conditions for the sub-functions (see, e.g., in [2]). These conditions ensure that the variance of $g(\boldsymbol{W})$ can also be partitioned into the sum of sub-variances:

$$
D \equiv \mathbb{E}\left[\left(g(\boldsymbol{W})-g_{0}\right)^{2}\right]=\sum_{i=1}^{M} D_{i}+\sum_{1 \leq i<j \leq M}^{M} D_{i j}+\cdots+D_{1,2, \ldots, M}
$$

with $D_{i_{1}, i_{2}, \ldots, i_{S}}$ denoting the contribution of the group of parameters $\left\{w_{i_{1}}, w_{i_{2}}, \ldots, w_{i_{S}}\right\}$. The Sobol' indices of each possible groups are defined via normalization with $D$,

$$
S_{i_{1}, i_{2}, \ldots, i_{S}}=D_{i_{1}, i_{2}, \ldots, i_{S}} / D
$$

that makes the sum of all possible indices equals to 1 . Indices corresponding to one setup parameter are called $1^{\text {st }}$ order indices. Higher order indices reflect the effect of the interaction of two or more setup parameters to the reconstruction uncertainty.

Polynomial chaos expansion (PCE). Even if surrogate models of $\boldsymbol{f}$ can effectively reduce the computational burden associated to Eq. (2), sensitivity analysis of $g(\boldsymbol{w})$ with respect to its input variables remains cumbersome when traditional methods (e.g., Monte Carlo sampling [7]) are used. Therefore, a PCE surrogate model of $g(\boldsymbol{w})$ is built, which provides robust estimates for the total variance $D$ and the Sobol' indices, respectively, with a moderate resource demand. The PCE approximation of each component of $\boldsymbol{g}$ reads as

$$
g(\boldsymbol{W}) \approx \sum_{\boldsymbol{\alpha} \in \mathscr{A}} c_{\boldsymbol{\alpha}} \Psi_{\boldsymbol{\alpha}}(\boldsymbol{W})
$$

where the basis functions $\Psi_{\boldsymbol{\alpha}}(\boldsymbol{W})$ are products of univariate functions, i.e., 


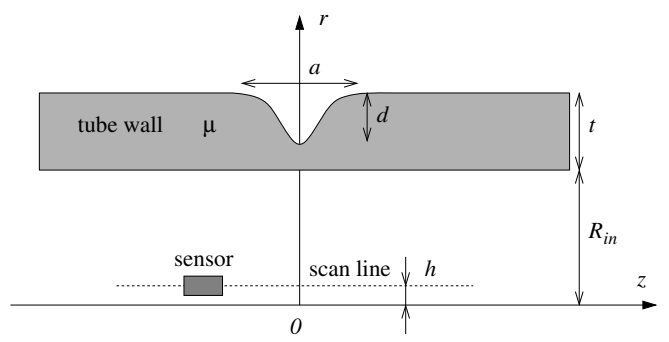

Figure 1. Sketch of the configuration: a ferromagnetic tube is affected by a circumferential groove on the exterior surface. A static magnetic field is incident in the axial (z) direction; the $z$-component of the leaking magnetic field is measured by a sensor scanning near the tube axis.

$$
\Psi_{\boldsymbol{\alpha}}(\boldsymbol{W})=\Phi_{\alpha_{1}}^{(1)}\left(W_{1}\right) \Phi_{\alpha_{2}}^{(2)}\left(W_{2}\right) \cdots \Phi_{\alpha_{M}}^{(M)}\left(W_{M}\right)
$$

where each factor $\Phi_{\alpha_{m}}^{(m)}$ is the $\alpha_{m}$-th member of a polynomial family which is orthonormal with respect to the marginal probability density function corresponding to the $m$-th setup parameter. For example, uniform and normal distributions correspond to Legendre and Hermite polynomials, respectively.

The index set $\mathscr{A}$ in Eq. (6) is chosen as a reasonable subset of $\mathbb{N}^{M}$, e.g., by a linear truncation $\mathscr{A}=\left\{\boldsymbol{\alpha}: \boldsymbol{\alpha} \in \mathbb{N}^{M},|\boldsymbol{\alpha}|_{1} \leq L\right\}$ where $L$ is a small integer (in our example, around 6...8). The coefficients $c_{\boldsymbol{\alpha}}$ in Eq. (6) are calculated by means of a least squares fit to a set of training data $\left\{\boldsymbol{w}^{(q)}, g\left(\boldsymbol{w}^{(q)}\right)\right\}, q=1,2, \ldots, Q$, obtained by $Q$ number of evaluations of Eq. (2). Once the PCE surrogate models for each component of $\boldsymbol{g}$ are built, the variance of $\boldsymbol{g}(\boldsymbol{W})$ and all Sobol' indices can be calculated from the PCE coefficients.

\section{Numerical examples}

The configuration and the EM model. A magnetic flux leakage NDE arrangement for tube inspection has been chosen as illustration. The configuration is kept simple so that the emphasis is put on the sensitivity analysis. A ferromagnetic tube (Fig. 1) with relative permeability $\mu$, inner radius $R_{\text {in }}$, and wall thickness $t$ is assumed to be affected by a circumferential groove on the exterior surface. An axially directed static magnetic field $\left(\boldsymbol{H}=\hat{z} H_{0}\right)$ is incident within the tube wall. Due to the groove, a leaking magnetic field is present. The total field is written as the sum of the incident field and the perturbation field caused by the groove: $\boldsymbol{H}=\boldsymbol{H}_{0}+\Delta \boldsymbol{H}$, where $\Delta \boldsymbol{H}$ has both axial and radial components. A sensor is scanning along a straight line parallel with the tube axis, and the $z$ component of the perturbation field $\left(\Delta H_{z}\right)$ is measured at 51 equi-spaced sensor positions, yielding the observable signal vector $\boldsymbol{y}$. The grove cross-section is modelled by a parametrized $3^{\text {rd }}$ order spline, with the parameters $a$ (opening) and $d$ (depth), denoted together as defect parameters $\boldsymbol{x}=[a, d]$. The region of interest in terms of defect parameters is defined by the ranges $a \in[0.5,1.5] \mathrm{mm}$ and $d \in[0.2,1.5] \mathrm{mm}$, respectively.

As uncertain setup parameters, the probe misalignment $h$, the tube wall thickness $t$, and the relative permeability $\mu$ are considered. They are modelled by independent random variables, with details on their distribution given in Table 1. The tube inner radius is kept fixed $\left(R_{\text {in }}=5 \mathrm{~mm}\right)$, and the length of the line scan is $20 \mathrm{~mm}$.

Due to the axial symmetry of the configuration, a two-dimensional EM model is applied. The perturbation field is calculated by means of the method of moments. 
Table 1. Probability distribution of the random setup parameters

\begin{tabular}{lll} 
parameter & distribution & properties \\
\hline probe misalignment $(h)$ & uniform & range $=[0,0.25] \mathrm{mm}$ \\
tube wall thickness $(t)$ & normal & mean $=2 \mathrm{~mm}$, standard deviation $=0.1 \mathrm{~mm}$ \\
tube relative permeability $(\mu)$ & normal & mean $=20$, standard deviation $=1.5($ Test A) or 0.5 (Test B)
\end{tabular}
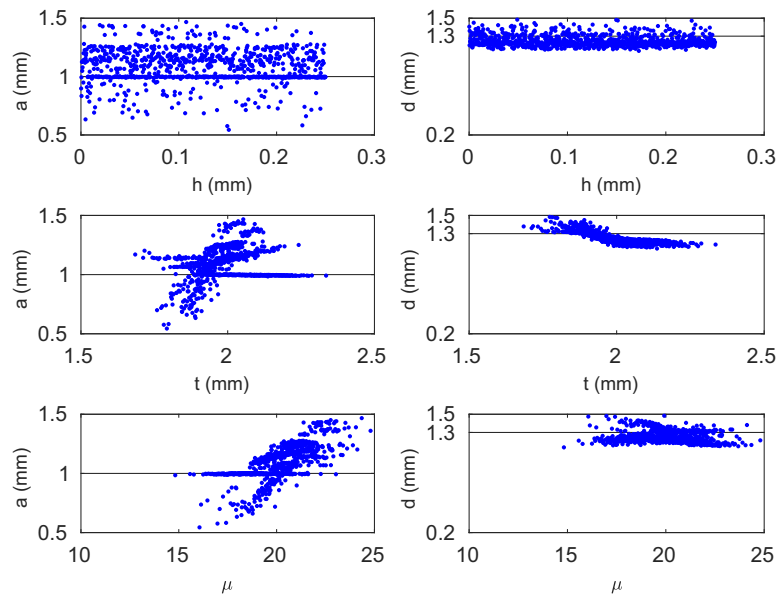

Figure 2. Distribution of the reconstructed defect parameters vs. realizations of the random setup parameters (legend: black line $[-]$ true parameter values; blue dots $[\cdot]$ reconstruction results)

Table 2. Defect parameters in the examples: true values and reconstruction results

\begin{tabular}{rrrrrr} 
& true & \multicolumn{2}{c}{ reconstructed mean } & \multicolumn{2}{c}{ standard deviation } \\
\hline & & Test A & Test B & Test A & Test B \\
$a(\mathrm{~mm})$ & 1.0 & 1.08 & 1.07 & 0.1460 & 0.1091 \\
$d(\mathrm{~mm})$ & 1.3 & 1.25 & 1.25 & 0.0611 & 0.0542
\end{tabular}

The optimization in Eq. (2) has been performed by the fmincon Matlabßfunction. One call of $\boldsymbol{f}$ and $\boldsymbol{g}$ (as in Eq. (2)) takes ca. $0.25 \mathrm{~s}$ and $10 \mathrm{~s}$, respectively, on a standard desktop PC (i7 CPU @ 3.60GHz, 8 GB RAM). The PCE surrogate models are built with $Q=900$ training samples in each case. Nevertheless, convergence studies subsequently proved that only 300 samples would have provided almost the same accuracy.

Studies and results. Two slightly different scenarios are studied. According to Table 1, the relative permeability of the tube wall has a higher (Test A) and a lower (Test B) variability. In both cases, the same defect is to be reconstructed: $[a, d]=[1.0,1.3] \mathrm{mm}$.

In Fig. 2, scatter plots present the reconstructed defect parameters wrt. the realization of the random setup parameters for Test A. Note that the uniform and normal distributions of the setup parameters (as defined in Table 1) are clearly seen, as well as that the reconstructed opening $a$ is sensitive to $t$ and $\mu$. Yet, the depth $d$ can be reconstructed with a high accuracy. Uncertainties are quantitatively characterized in Table 2.

The sources of the uncertainties are ranked by means of Sobol' indices (Fig. 3). The bar plots are in harmony with the conclusions of Fig. 2: the probe misalignment $h$ 


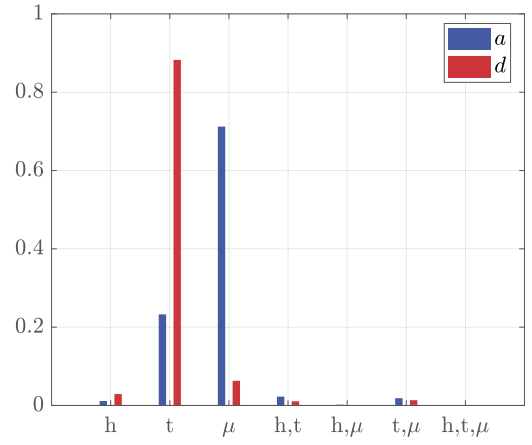

(a) Test A

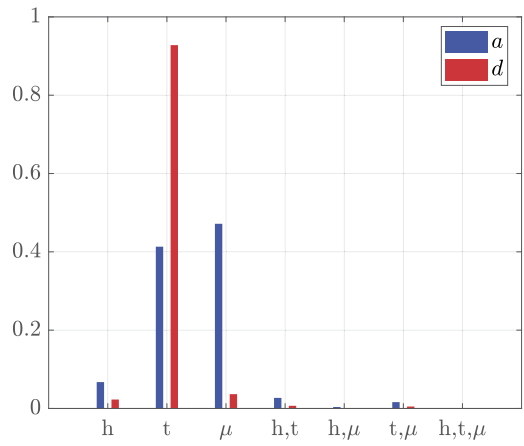

(b) Test B

Figure 3. Sobol' indices.

has little effect to the reconstruction. On the contrary, the effect of the uncertainty of $t$ and $\mu$ is significant. When changing from Test $\mathrm{A}$ to $\mathrm{B}$ (i.e., $\mu$ is better controlled), the Sobol' index of $\mu$ decreases as expected. Mutual effects ( $2^{\text {nd }}$ and $3^{\text {rd }}$ order indices) in this example are actually not significant, yet this method is able to reveal such interactions.

\section{Conclusion}

A framework is presented for the sensitivity analysis of model-based inverse problems of EM NDE with respect to uncertain setup parameters. The computation burden that is commonly associated to inverse problems, can be reduced by surrogate models. The entire inversion scheme is approximated by a polynomial chaos expansion surrogate model, that provides a simple way to calculate Sobol' indices. In so doing, the sources of uncertainty can be ranked based on their importance, which reveals the most efficient ways of improving the accuracy of the NDE arrangement. A simple magnetic flux leakage NDE method has been analysed to demonstrate the capabilities of the framework.

\section{References}

[1] Sobol' IM. Sensitivity estimates for nonlinear mathematical models. Mathematical Modelling and Computational Experiments. 1993;1(4):407-414.

[2] Sudret B. Global sensitivity analysis using polynomial chaos expansions. Reliability Engineering \& System Safety. 2008;93(7):964-979.

[3] Zhu P, Cheng Y, Banerjee P, Tamburrino A, Deng Y. A novel machine learning model for eddy current testing with uncertainty. NDT \& E International. 2019;101:104 - 112.

[4] Bingler A, Bilicz S. Sensitivity Analysis Using a Sparse Grid Surrogate Model in Electromagnetic NDE. In: Lesselier D, Reboud C, editors. Electromagnetic Non-Destructive Evaluation (XXI). vol. 43 of Studies in Applied Electromagnetics and Mechanics. IOS Press; 2018. p. 152-159.

[5] Bilicz S. Sparse grid surrogate models for electromagnetic problems with many parameters. IEEE Transactions on Magnetics. 2016;52(3):1-4.

[6] Douvenot R, Lambert M, Lesselier D. Adaptive Metamodels for Crack Characterization in Eddy-Current Testing. IEEE Transactions on Magnetics. 2011;47(4):746-755.

[7] Fan M, Wu G, Cao B, Sarkodie-Gyan T, Li Z, Tian G. Uncertainty metric in model-based eddy current inversion using the adaptive Monte Carlo method. Measurement. 2019;137:323 - 331. 